\title{
FABER POLYNOMIAL COEFFICIENT ESTIMATES FOR BI-UNIVALENT FUNCTIONS OF COMPLEX ORDER BASED ON SUBORDINATE CONDITIONS INVOLVING OF THE JACKSON $(p, q)$-DERIVATIVE
}

\author{
ŞAHSENE ALTINKAYA AND SIBEL YALÇIN
}

Received 12 January, 2017

\begin{abstract}
In the present paper, new subclasses of bi-univalent functions of complex order associated with the $(p, q)$-derivative are introduced. Furthermore, using the Faber polynomial expansions, we get upper bounds for the coefficients of functions belonging to these classes.
\end{abstract}

2010 Mathematics Subject Classification: 30C45; 30C80; 33D15

Keywords: bi-univalent functions, Faber polynomials, $(p, q)$-derivative operator

\section{INTRODUCTION, DEFINITIONS AND NOTATIONS}

Denote by $E$ the unit disc of the complex plane, $E=\{z: z \in \mathbb{C}$ and $|z|<1\}, A$ the class of functions analytic in $E$, satisfying the conditions

$$
f(0)=0 \text { and } f^{\prime}(0)=1 .
$$

Then each function $f$ in $A$ has the Taylor expansion

$$
f(z)=z+\sum_{n=2}^{\infty} a_{n} z^{n} .
$$

We denote by $S$ the subclass of $A$ consisting of functions the form (1.1) which are also univalent in $E$.

For $f$ and $F$ analytic in $E$, we say that $f$ is subordinate to $F$, written $f \prec F$, if there exists a Schwarz function

$$
t(z)=\sum_{n=1}^{\infty} c_{n} z^{n}
$$

with $|t(z)|<1$ in $E$, such that $f(z)=F(t(z))$. For the Schwarz function $t(z)$ we note that $\left|c_{n}\right|<1$. (e.g. see Duren [11]). 
It is well known that every function $f \in S$ has an inverse $f^{-1}$, satisfying $f^{-1}(f(z))=z(z \in E)$ and $f\left(f^{-1}(w)\right)=w\left(|w|<r_{0}(f), r_{0}(f) \geq \frac{1}{4}\right)$, where

$$
f^{-1}(w)=w-a_{2} w^{2}+\left(2 a_{2}^{2}-a_{3}\right) w^{3}-\left(5 a_{2}^{3}-5 a_{2} a_{3}+a_{4}\right) w^{4}+\cdots .
$$

A function $f \in A$ is said to be bi-univalent in $E$ if both $f$ and $f^{-1}$ are univalent in $E$. Let $\Sigma$ denote the class of bi-univalent functions in $E$ given by (1.1). For a brief history and interesting examples in the class $\Sigma$, see [24] (see also [7], [8], [19], [20]). Furthermore, judging by the remarkable flood of papers on the subject (see, for example, [9], [5], [17], [23], [24], [25]).

The Faber polynomials introduced by Faber [12] play an important role in various areas of mathematical sciences, especially in Geometric Function Theory. Grunsky [14] succeeded in establishing a set of conditions for a given function which are necessary and in their totality sufficient for the univalency of this function, and in these conditions the coefficients of the Faber polynomials play an important role. Schiffer [22] gave a differential equations for univalent functions solving certain extremum problems with respect to coefficients of such functions; in this differential equation appears again a polynomial which is just the derivative of a Faber polynomial (Schaeffer-Spencer [21]).

Not much is known about the bounds on the general coefficient $\left|a_{n}\right|$. In the literature, there are only a few works determining the general coefficient bounds $\left|a_{n}\right|$ for the analytic bi-univalent functions ([6], [15], [16]). The coefficient estimate problem for each of $\left|a_{n}\right|(n \in \mathbb{N} \backslash\{1,2\} ; \mathbb{N}=\{1,2,3, \ldots\})$ is still an open problem.

\subsection{The Jackson $(p, q)$-derivative}

For the convenience, we provide some basic definitions and concept details of $q$ calculus which are used in this paper. We suppose throughout the paper that $0<q<$ $p \leq 1$. We recall the definitions of fractional $q$-calculus operators of complex valued function $f$. We shall follow the notation and terminology in [13].

Definition 1 (see [10]). The $(p, q)$-derivative of the function $f$ is defined as

$$
\left(D_{p, q} f\right)(z)=\left\{\begin{array}{cc}
\frac{f(p z)-f(q z)}{(p-q) z} & \text { for } z \neq 0 \\
f^{\prime}(0) & \text { for } z=0
\end{array} .\right.
$$

From (1.3), we deduce that

$$
\left(D_{p, q} f\right)(z)=1+\sum_{n=2}^{\infty}[n]_{p, q} a_{n} z^{n-1},
$$

where the symbol $[n]_{p, q}$ denotes the so-called $(p, q)$-bracket or twin-basic number

$$
[n]_{p, q}=\frac{p^{n}-q^{n}}{p-q} .
$$


It happens clearly that $D_{p, q} z^{n}=[n]_{p, q} z^{n-1}$. Note also that for $p=1$, the Jackson $(p, q)$-derivative reduces to the Jackson $q$-derivative given by (see [18])

$$
\left(D_{q} f\right)(z)=\frac{f(z)-f(q z)}{(1-q) z}, \quad z \neq 0 .
$$

The twin-basic number is a natural generalization of the $q$-number, that is

$$
\lim _{p \rightarrow 1}[n]_{p, q}=[n]_{q}=\frac{1-q^{n}}{1-q}, q \neq 1 .
$$

As with ordinary derivative, the action of the $(p, q)$-derivative of a function is a linear operator. More precisely, for any constants $a$ and $b$,

$$
D_{p, q}(a f(z)+b h(z))=a D_{p, q} f(z)+b D_{p, q} h(z) .
$$

The $(p, q)$-derivative fulfils the following product rules

$$
\begin{aligned}
& D_{p, q}(f(z) h(z))=f(p z) D_{p, q} h(z)+h(q z) D_{p, q} f(z), \\
& D_{p, q}(f(z) h(z))=h(p z) D_{p, q} f(z)+f(q z) D_{p, q} h(z) .
\end{aligned}
$$

Further, the $(p, q)$-derivative fulfils the following product rules

$$
\begin{gathered}
D_{p, q}\left(\frac{f(z)}{h(z)}\right)=\frac{h(q z) D_{p, q} f(z)-f(q z) D_{p, q} h(z)}{h(p z) h(q z)}, \\
D_{p, q}\left(\frac{f(z)}{h(z)}\right)=\frac{h(p z) D_{p, q} f(z)-f(p z) D_{p, q} h(z)}{h(p z) h(q z)} .
\end{gathered}
$$

From (1.2) and (1.3), we also deduce that

$$
\begin{aligned}
\left(D_{p, q} g\right)(w) & =\frac{g(p w)-g(q w)}{(p-q) w} \\
& =1-[2]_{p, q} a_{2} w+[3]_{p, q}\left(2 a_{2}^{2}-a_{3}\right) w^{2} \\
& -[4]_{p, q}\left(5 a_{2}^{3}-5 a_{2} a_{3}+a_{4}\right) w^{3}+\cdots
\end{aligned}
$$

where the function $g$ is given by (1.2).

\section{PRELIMINARY RESUlTS}

By using the Faber polynomial expansion of functions $f \in A$ of the form (1.1), the coefficients of its inverse map $g=f^{-1}$ may be expressed as follows (see [3]):

$$
g(w)=f^{-1}(w)=w+\sum_{n=2}^{\infty} \frac{1}{n} K_{n-1}^{-n}\left(a_{2}, a_{3}, \ldots\right) w^{n},
$$

where

$$
K_{n-1}^{-n}=\frac{(-n) !}{(-2 n+1) !(n-1) !} a_{2}^{n-1}+\frac{(-n) !}{[2(-n+1)] !(n-3) !} a_{2}^{n-3} a_{3}
$$




$$
\begin{array}{r}
+\frac{(-n) !}{(-2 n+3) !(n-4) !} a_{2}^{n-4} a_{4} \\
+\frac{(-n) !}{[2(-n+2)] !(n-5) !} a_{2}^{n-5}\left[a_{5}+(-n+2) a_{3}^{2}\right] \\
+\frac{(-n) !}{(-2 n+5) !(n-6) !} a_{2}^{n-6}\left[a_{6}+(-2 n+5) a_{3} a_{4}\right] \\
+\sum_{j \geq 7} a_{2}^{n-j} V_{j},
\end{array}
$$

such that $V_{j}$ with $7 \leq j \leq n$ is a homogeneous polynomial in the variables $a_{2}, a_{3}, \ldots, a_{n}$ [4]. In particular, the first three terms of $K_{n-1}^{-n}$ are given below:

$$
\begin{array}{r}
\frac{1}{2} K_{1}^{-2}=-a_{2}, \\
\frac{1}{3} K_{2}^{-3}=2 a_{2}^{2}-a_{3}, \\
\frac{1}{4} K_{3}^{-4}=-\left(5 a_{2}^{3}-5 a_{2} a_{3}+a_{4}\right) .
\end{array}
$$

In general, for any $p \in \mathbb{N}$ and $n \geq 2$, an expansion of $K_{n-1}^{p}$ is as, [3],

$$
K_{n-1}^{p}=p a_{n}+\frac{p(p-1)}{2} E_{n-1}^{2}+\frac{p !}{(p-3) ! 3 !} E_{n-1}^{3}+\ldots+\frac{p !}{(p-n+1) !(n-1) !} E_{n-1}^{n-1},
$$

where $E_{n-1}^{p}=E_{n-1}^{p}\left(a_{2}, a_{3}, \ldots\right)$ and by [1],

$$
E_{n-1}^{m}\left(a_{2}, \ldots, a_{n}\right)=\sum_{n=2}^{\infty} \frac{m !\left(a_{2}\right)^{\mu_{1}} \ldots\left(a_{n}\right)^{\mu_{n-1}}}{\mu_{1} ! \ldots \mu_{n-1} !}, \quad \text { for } m \leq n
$$

while $a_{1}=1$, and the sum is taken over all nonnegative integers $\mu_{1}, \ldots, \mu_{n}$ satisfying

$$
\begin{array}{r}
\mu_{1}+\mu_{2}+\ldots+\mu_{n-1}=m, \\
\mu_{1}+2 \mu_{2}+\ldots+(n-1) \mu_{n-1}=n-1 .
\end{array}
$$

Evidently, $E_{n-1}^{n-1}\left(a_{2}, \ldots, a_{n}\right)=a_{2}^{n-1}$,(see [2]); while $a_{1}=1$, and the sum is taken over all nonnegative integers $\mu_{1}, \ldots, \mu_{n}$ satisfying

$$
\begin{array}{r}
\mu_{1}+\mu_{2}+\ldots+\mu_{n}=m, \\
\mu_{1}+2 \mu_{2}+\ldots+n \mu_{n}=n .
\end{array}
$$

It is clear that $E_{n}^{n}\left(a_{1}, a_{2}, \ldots, a_{n}\right)=a_{1}^{n}$. The first and the last polynomials are:

$$
E_{n}^{1}=a_{n}, \quad E_{n}^{n}=a_{1}^{n} \text {. }
$$


In the following, let $\phi$ be an analytic function with positive real part in $E$, with $\phi(0)=1$ and $\phi^{\prime}(0)>0$. Also, let $\phi(E)$ be starlike with respect to 1 and symmetric with respect to the real axis. Thus, $\phi$ has the Taylor series expansion

$$
\phi(z)=1+B_{1} z+B_{2} z^{2}+B_{3} z^{3}+\cdots \quad\left(B_{1}>0\right) .
$$

Suppose that $u(z)$ and $v(w)$ are analytic in the unit disk $E$ with $u(0)=v(0)=$ $0,|u(z)|<1,|v(w)|<1$, and suppose that

$$
u(z)=p_{1} z+\sum_{n=2}^{\infty} p_{n} z^{n}, v(w)=q_{1} w+\sum_{n=2}^{\infty} q_{n} w^{n} \quad(|z|<1) .
$$

It is well known that

$$
\left|p_{1}\right| \leq 1,\left|p_{2}\right| \leq 1-\left|p_{1}\right|^{2},\left|q_{1}\right| \leq 1,\left|q_{2}\right| \leq 1-\left|q_{1}\right|^{2} .
$$

Next, the equations (2.4) and (2.5) lead to

$$
\begin{aligned}
\phi(u(z)) & =1+B_{1} u(z)+B_{2}(u(z))^{2} z^{2}+\cdots \\
& =1+B_{1} p_{1} z+\left(B_{1} p_{2}+B_{2} p_{1}^{2}\right) z^{2}+\cdots \\
& =1+\sum_{n=1}^{\infty} \sum_{k=1}^{n} B_{k} E_{n}^{k}\left(p_{1}, p_{2}, \ldots, p_{n}\right) z^{n}
\end{aligned}
$$

and

$$
\begin{aligned}
\phi(v(w)) & =1+B_{1} v(w)+B_{2}(v(w))^{2} z^{2}+\cdots \\
& =1+B_{1} q_{1} w+\left(B_{1} q_{2}+B_{2} q_{1}^{2}\right) w^{2}+\cdots \\
& =1+\sum_{n=1}^{\infty} \sum_{k=1}^{n} B_{k} E_{n}^{k}\left(q_{1}, q_{2}, \ldots, q_{n}\right) w^{n}
\end{aligned}
$$

Definition 2. A function $f \in \Sigma$ is said to be in the class $R_{\Sigma, b}^{p, q}(\phi)$ if the following subordination relationships hold true:

$$
\left[1+\frac{1}{b}\left(\left(D_{p, q} f\right)(z)-1\right)\right] \prec \phi(z)
$$

and

$$
\left[1+\frac{1}{b}\left(\left(D_{p, q} g\right)(w)-1\right)\right] \prec \phi(w)
$$


where $b \in \mathbb{C} \backslash\{0\}, 0<q<p \leq 1 ; z, w \in E$ and the function $g$ is given by (1.2).

We note from Definition 2 that

$\lim _{p \rightarrow 1} R_{\Sigma, b}^{p, q}(\phi)=\left\{f \in \Sigma:\left\{\begin{array}{l}\lim _{p \rightarrow 1}\left[1+\frac{1}{b}\left(\left(D_{p, q} f\right)(z)-1\right)\right] \prec \phi(z) \\ \lim _{p \rightarrow 1}\left[1+\frac{1}{b}\left(\left(D_{p, q} g\right)(w)-1\right)\right] \prec \phi(w)\end{array}\right\}=R_{\Sigma, b}^{q}(\phi)\right.$.

By suitably specializing the parameters $b$ and $p$, we state the new subclasses of bi-univalent functions of complex order $R_{\Sigma, b}^{p, q}(\phi)$ as illustrated in the following Definitions.

Definition 3. For $b=1$, a function $f \in \Sigma$ is said to be in the class $R_{\Sigma}^{p, q}(\phi)$ if it satisfies the following conditions respectively:

$$
\left(D_{p, q} f\right)(z) \prec \phi(z)
$$

and

$$
\left(D_{p, q} g\right)(w) \prec \phi(w)
$$

where $0<q<p \leq 1 ; z, w \in E$ and the function $g$ is given by (1.2).

Definition 4. For $b=1$ and $p \rightarrow 1$, a function $f \in \Sigma$ is said to be in the class $R_{\Sigma}^{q}(\phi)$ if it satisfies the following conditions respectively:

$$
\left(D_{q} f\right)(z) \prec \phi(z)
$$

and

$$
\left(D_{q} g\right)(w) \prec \phi(w)
$$

where $z, w \in E$ and the function $g$ is given by (1.2).

In this paper, we study the class $R_{\Sigma, b}^{p, q}(\phi)$ of analytic bi-univalent functions defined above by using the $(p, q)$-derivative operator. Moreover, we use the Faber polynomial expansions to obtain bounds for the general coefficients $\left|a_{n}\right|$ of bi-univalent functions in $R_{\Sigma, b}^{p, q}(\phi)$ as well as we provide estimates for the initial coefficients of these functions. Several new consequences of the results are also pointed out.

\section{MAIN RESULTS}

Our first main result is given by Theorem 1 below.

Theorem 1. For $b \in \mathbb{C} \backslash\{0\}$, let $f \in \mathrm{R}_{\Sigma, b}^{p, q}(\phi)$. If $a_{m}=0 ; 2 \leq m \leq n-1$, then

$$
\left|a_{n}\right| \leq \frac{B_{1}|b|}{[n]_{p, q}}, \quad n \geq 3 .
$$


Proof. Let $f$ be given by (1.1). We have

$$
\left(D_{p, q} f\right)(z)-1=\sum_{n=2}^{\infty}[n]_{p, q} a_{n} z^{n-1},
$$

and, for its inverse map $g=f^{-1}$, it is seen that

$$
\left(D_{p, q} g\right)(w)-1=\sum_{n=2}^{\infty} K_{n-1}^{-n}\left(a_{2}, a_{3}, \ldots\right) w^{n-1}=\sum_{n=2}^{\infty}[n]_{p, q} b_{n} w^{n-1} .
$$

From (2.9) and (2.10) yields

$$
1+\frac{1}{b}\left(\left(D_{p, q} f\right)(z)-1\right)=\varphi(u(z))
$$

and

$$
1+\frac{1}{b}\left(\left(D_{p, q} g\right)(w)-1\right)=\varphi(v(w))
$$

Comparing the corresponding coefficients of (3.3) and (3.4) yields

$$
\frac{1}{b}[n]_{p, q} a_{n}=B_{1} p_{n-1},
$$

and

$$
\frac{1}{b}[n]_{p, q} b_{n}=B_{1} q_{n-1} .
$$

Note that for $a_{m}=0 ; 2 \leq m \leq n-1$ we have $b_{n}=-a_{n}$ and so

$$
\begin{aligned}
\frac{1}{b}[n]_{p, q} a_{n} & =B_{1} p_{n-1}, \\
-\frac{1}{b}[n]_{p, q} a_{n} & =B_{1} q_{n-1} .
\end{aligned}
$$

Now taking the absolute values of either of the above two equations and from (2.6), we obtain

$$
\left|a_{n}\right|=\frac{B_{1}\left|p_{n-1}\right||b|}{[n]_{p, q}}=\frac{B_{1}\left|q_{n-1}\right||b|}{[n]_{p, q}} \leq \frac{B_{1}|b|}{[n]_{p, q}} .
$$

Theorem 2. For $b \in \mathbb{C} \backslash\{0\}$, let $f \in \mathrm{R}_{\Sigma, b}^{q}(\phi)$. If $a_{m}=0 ; 2 \leq m \leq n-1$, then

$$
\left|a_{n}\right| \leq \frac{B_{1}|b|(1-q)}{1-q^{n}} ; \quad n \geq 3 .
$$

Theorem 3. Let $f \in \mathrm{R}_{\Sigma}^{p, q}(\phi)$. If $a_{m}=0 ; 2 \leq m \leq n-1$, then

$$
\left|a_{n}\right| \leq \frac{B_{1}}{[n]_{p, q}} ; \quad n \geq 3 .
$$


Theorem 4. Let $f \in \mathrm{R}_{\Sigma}^{q}(\phi)$. If $a_{m}=0 ; 2 \leq m \leq n-1$, then

$$
\left|a_{n}\right| \leq \frac{B_{1}(1-q)}{1-q^{n}} ; \quad n \geq 3 .
$$

\section{Coefficient estimates}

In this section we obtain coefficient estimates for functions belonging to the classes $R_{\Sigma, b}^{p, q}(\phi), R_{\Sigma, b}^{q}(\phi), R_{\Sigma}^{p, q}(\phi)$ and $R_{\Sigma}^{q}(\phi)$.

Theorem 5. Let $f \in \mathrm{R}_{\Sigma, b}^{p, q}(\phi)(b \in \mathbb{C} \backslash\{0\})$. Then

$\left|a_{2}\right| \leq \min \left\{K(p, q), \frac{|b| B_{1} \sqrt{B_{1}}}{\sqrt{\left|\left(B_{1}^{2} b-B_{2}\right)\left(p^{2}+q^{2}\right)+\left(B_{1}^{2} b-2 B_{2}\right) p q\right|+B_{1}\left(p^{2}+2 p q+q^{2}\right)}}\right\}$ and

$$
\left|a_{3}\right| \leq \min \{L(p, q), M(p, q)\}
$$

where

and

$$
\begin{gathered}
K(p, q)= \begin{cases}\sqrt{\frac{B_{1}|b|}{p^{2}+p q+q^{2}} ;} & \left|B_{2}\right| \leq B_{1} \\
\sqrt{\frac{\left|B_{2}\right||b|}{p^{2}+p q+q^{2}} ;} & \left|B_{2}\right|>B_{1}\end{cases} \\
L(p, q)= \begin{cases}\frac{B_{1}|b|}{p^{2}+p q+q^{2}} ; & \left|B_{2}\right| \leq B_{1} \\
\frac{\left|B_{2}\right||b|}{p^{2}+p q+q^{2}} ; & \left|B_{2}\right|>B_{1}\end{cases}
\end{gathered}
$$

$$
M(p, q)=\left\{\begin{array}{cc}
\frac{B_{1}|b|}{p^{2}+p q+q^{2}} ; & B_{1} \leq \frac{p^{2}+2 p q+q^{2}}{\left(p^{2}+p q+q^{2}\right)|b|} \\
\frac{|b| B_{1}\left[\left|\left(B_{1}^{2} b-B_{2}\right)\left(p^{2}+q^{2}\right)+\left(B_{1}^{2} b-2 B_{2}\right) p q\right|+B_{1}^{2}|b|\left(p^{2}+p q+q^{2}\right)\right]}{\left[\left|\left(B_{1}^{2} b-B_{2}\right)\left(p^{2}+q^{2}\right)+\left(B_{1}^{2} b-2 B_{2}\right) p q\right|+B_{1}\left(p^{2}+2 p q+q^{2}\right)\right]\left(p^{2}+p q+q^{2}\right)} & B_{1}>\frac{p^{2}+2 p q+q^{2}}{\left(p^{2}+p q+q^{2}\right)|b|} .
\end{array}\right.
$$

Proof. Replacing $n$ by 2 and 3 in (3.5) and (3.6), respectively, we find that

$$
\begin{gathered}
\frac{1}{b}[2]_{p, q} a_{2}=B_{1} p_{1}, \\
\frac{1}{b}[3]_{p, q} a_{3}=B_{1} p_{2}+B_{2} p_{1}^{2}, \\
-\frac{1}{b}[2]_{p, q} a_{2}=B_{1} q_{1}, \\
\frac{1}{b}[3]_{p, q}\left(2 a_{2}^{2}-a_{3}\right)=B_{1} q_{2}+B_{2} q_{1}^{2} .
\end{gathered}
$$


From (4.1) and (4.3), we obtain

$$
p_{1}=-q_{1}
$$

By adding (4.4) to (4.2), further computations using (4.5) lead to

$$
\frac{2}{b}[3]_{p, q} a_{2}^{2}=B_{1}\left(p_{2}+q_{2}\right)+2 B_{2} p_{1}^{2} .
$$

Making use of (4.1) in the above equality (4.6), we get

$$
\left[2 b B_{1}^{2}[3]_{p, q}-2 B_{2}[2]_{p, q}^{2}\right] a_{2}^{2}=b^{2} B_{1}^{3}\left(p_{2}+q_{2}\right) .
$$

Combining (4.7) and (2.6), we obtain

$$
\begin{aligned}
2\left|b B_{1}^{2}[3]_{p, q}-B_{2}[2]_{p, q}^{2}\right|\left|a_{2}\right|^{2} & \leq|b|^{2} B_{1}^{3}\left(\left|p_{2}\right|+\left|q_{2}\right|\right) \\
& \leq 2|b|^{2} B_{1}^{3}\left(1-\left|p_{1}\right|^{2}\right) \\
& =2|b|^{2} B_{1}^{3}-2|b|^{2} B_{1}^{3}\left|p_{1}\right|^{2}
\end{aligned}
$$

It follows from (4.1) that

$$
\left|a_{2}\right| \leq \frac{|b| B_{1} \sqrt{B_{1}}}{\sqrt{\left|B_{1}^{2} b[3]_{p, q}-B_{2}[2]_{p, q}^{2}\right|+B_{1}[2]_{p, q}^{2}}}
$$

Moreover, by (2.6) and (4.6)

$$
\begin{aligned}
\frac{2}{|b|}[3]_{p, q}\left|a_{2}\right|^{2} & \leq B_{1}\left(\left|p_{2}\right|+\left|q_{2}\right|\right)+2\left|B_{2}\right|\left|p_{1}\right|^{2} \\
& \leq 2 B_{1}\left(1-\left|p_{1}\right|^{2}\right)+2\left|B_{2}\right|\left|p_{1}\right|^{2} \\
& =2 B_{1}+2\left|p_{1}\right|^{2}\left(\left|B_{2}\right|-B_{1}\right) \\
\frac{1}{|b|}[3]_{p, q}\left|a_{2}\right|^{2} & \leq\left\{\begin{array}{c}
B_{1} ;\left|B_{2}\right| \leq B_{1} \\
\left|B_{2}\right| ;\left|B_{2}\right|>B_{1}
\end{array}\right.
\end{aligned}
$$


Clearly, we can see that

$$
\left|a_{2}\right| \leq\left\{\begin{array}{ll}
\sqrt{\frac{B_{1}|b|}{[3]_{p, q}} ;} & \left|B_{2}\right| \leq B_{1} \\
\sqrt{\frac{\left|B_{2}\right||b|}{[3]_{p, q}} ;} & \left|B_{2}\right|>B_{1}
\end{array} .\right.
$$

Next, in order to find the bound on $\left|a_{3}\right|$, by subtracting (4.4) from (4.2), we obtain

$$
\frac{2}{b}[3]_{p, q} a_{3}=\frac{2}{b}[3]_{p, q} a_{2}^{2}+B_{1}\left(p_{2}-q_{2}\right) .
$$

Clearly, from (4.6), we have that

$$
\begin{aligned}
a_{3} & =\frac{b\left[B_{1}\left(p_{2}+q_{2}\right)+2 B_{2} p_{1}^{2}\right]}{2[3]_{p, q}}+\frac{b B_{1}\left(p_{2}-q_{2}\right)}{2[3]_{p, q}} \\
& =\frac{b B_{1} p_{2}+b B_{2} p_{1}^{2}}{[3]_{p, q}}
\end{aligned}
$$

and consequently

$$
\begin{aligned}
\left|a_{3}\right| & \leq \frac{|b| B_{1}\left|p_{2}\right|+|b|\left|B_{2}\right|\left|p_{1}\right|^{2}}{[3]_{p, q}} \\
& \leq \frac{|b| B_{1}\left(1-\left|p_{1}\right|^{2}\right)+|b|\left|B_{2}\right|\left|p_{1}\right|^{2}}{[3]_{p, q}} \\
& =\frac{|b| B_{1}+|b|\left|p_{1}\right|^{2}\left(\left|B_{2}\right|-B_{1}\right)}{[3]_{p, q}} .
\end{aligned}
$$

Hence, we write

$$
\left|a_{3}\right| \leq \begin{cases}\frac{B_{1}|b|}{[3]_{p, q} ;} & \left|B_{2}\right| \leq B_{1} \\ \frac{\left|B_{2}\right||b|}{[3]_{p, q} ;} & \left|B_{2}\right|>B_{1} .\end{cases}
$$

On the other hand, by using (2.6) and (4.10), we have

$$
\begin{aligned}
\frac{2}{|b|}[3]_{p, q}\left|a_{3}\right| & \leq \frac{2}{|b|}[3]_{p, q}\left|a_{2}\right|^{2}+B_{1}\left(\left|p_{2}\right|+\left|q_{2}\right|\right) \\
& \leq \frac{2}{|b|}[3]_{p, q}\left|a_{2}\right|^{2}+2 B_{1}\left(1-\left|p_{1}\right|^{2}\right) .
\end{aligned}
$$


Then, with the help of (4.1), we have

$$
B_{1}|b|[3]_{p, q}\left|a_{3}\right| \leq\left[B_{1}|b|[3]_{p, q}-[2]_{p, q}^{2}\right]\left|a_{2}\right|^{2}+B_{1}^{2}|b|^{2} .
$$

Now, from (4.9), we obtain

$$
\left|a_{3}\right| \leq \frac{B_{1}|b|}{[3]_{p, q}}\left\{1+\frac{B_{1}\left[B_{1}|b|[3]_{p, q}-[2]_{p, q}^{2}\right]}{\left|B_{1}^{2} b[3]_{p, q}-B_{2}[2]_{p, q}^{2}\right|+B_{1}[2]_{p, q}^{2}}\right\} .
$$

Theorem 6. Let $f \in \mathrm{R}_{\Sigma, b}^{q}(\phi)(b \in \mathbb{C} \backslash\{0\})$. Then

$$
\left|a_{2}\right| \leq \min \left\{K(q), \frac{|b| B_{1} \sqrt{B_{1}}}{\sqrt{\left|\left(B_{1}^{2} b-B_{2}\right)\left(1+q^{2}\right)+\left(B_{1}^{2} b-2 B_{2}\right) q\right|+B_{1}\left(1+2 q+q^{2}\right)}}\right\}
$$

and

$$
\left|a_{3}\right| \leq \min \{L(q), M(q)\}
$$

where

$$
\begin{gathered}
K(q)=\left\{\begin{array}{ll}
\sqrt{\frac{B_{1}|b|}{1+q+q^{2}} ;} & \left|B_{2}\right| \leq B_{1} \\
\sqrt{\frac{\left|B_{2}\right||b|}{1+q+q^{2}} ;} & \left|B_{2}\right|>B_{1}
\end{array},\right. \\
L(q)= \begin{cases}\frac{B_{1}|b|}{1+q+q^{2}} ; & \left|B_{2}\right| \leq B_{1} \\
\frac{\left|B_{2}\right||b|}{1+q+q^{2}} ; & \left|B_{2}\right|>B_{1}\end{cases}
\end{gathered}
$$

and

$$
M(q)=\left\{\begin{array}{ll}
\frac{B_{1}|b|}{1+q+q^{2}} ; & B_{1} \leq \frac{1+2 q+q^{2}}{\left(1+q+q^{2}\right)|b|} \\
\frac{|b| B_{1}\left[\left|\left(B_{1}^{2} b-B_{2}\right)\left(1+q^{2}\right)+\left(B_{1}^{2} b-2 B_{2}\right) q\right|+B_{1}^{2}|b|\left(1+q+q^{2}\right)\right]}{\left[\left|\left(B_{1}^{2} b-B_{2}\right)\left(1+q^{2}\right)+\left(B_{1}^{2} b-2 B_{2}\right) q\right|+B_{1}\left(1+2 q+q^{2}\right)\right]\left(1+q+q^{2}\right)} ; & B_{1}>\frac{1+2 q+q^{2}}{\left(1+q+q^{2}\right)|b|}
\end{array} .\right.
$$

Theorem 7. Let $f \in \mathrm{R}_{\Sigma}^{p, q}(\phi)$. Then

$$
\left|a_{2}\right| \leq \min \left\{K(p, q), \frac{B_{1} \sqrt{B_{1}}}{\sqrt{\left|\left(B_{1}^{2}-B_{2}\right)\left(p^{2}+q^{2}\right)+\left(B_{1}^{2}-2 B_{2}\right) p q\right|+B_{1}\left(p^{2}+2 p q+q^{2}\right)}}\right\}
$$

and

$$
\left|a_{3}\right| \leq \min \{L(p, q), M(p, q)\}
$$


where

$$
\begin{gathered}
K(p, q)=\left\{\begin{array}{ll}
\sqrt{\frac{B_{1}}{p^{2}+p q+q^{2}} ;} & \left|B_{2}\right| \leq B_{1} \\
\sqrt{\frac{\left|B_{2}\right|}{p^{2}+p q+q^{2}} ;} & \left|B_{2}\right|>B_{1}
\end{array},\right. \\
L(p, q)= \begin{cases}\frac{B_{1}}{p^{2}+p q+q^{2}} ; & \left|B_{2}\right| \leq B_{1} \\
\frac{\left|B_{2}\right|}{p^{2}+p q+q^{2}} ; & \left|B_{2}\right|>B_{1}\end{cases}
\end{gathered}
$$

and

$$
M(p, q)=\left\{\begin{array}{ll}
\frac{B_{1}}{p^{2}+p q+q^{2}} ; & B_{1} \leq \frac{p^{2}+2 p q+q^{2}}{p^{2}+p q+q^{2}} \\
\frac{B_{1}\left[\left|\left(B_{1}^{2}-B_{2}\right)\left(p^{2}+q^{2}\right)+\left(B_{1}^{2}-2 B_{2}\right) p q\right|+B_{1}^{2}\left(p^{2}+p q+q^{2}\right)\right]}{\left[\left(B_{1}^{2}-B_{2}\right)\left(p^{2}+q^{2}\right)+\left(B_{1}^{2}-2 B_{2}\right) p q \mid+B_{1}\left(p^{2}+2 p q+q^{2}\right)\right]\left(p^{2}+p q+q^{2}\right)} ; & B_{1}>\frac{p^{2}+2 p q+q^{2}}{p^{2}+p q+q^{2}}
\end{array} .\right.
$$

Theorem 8. Let $f \in \mathrm{R}_{\Sigma}^{q}(\phi)$. Then

$$
\left|a_{2}\right| \leq \min \left\{K(q), \frac{B_{1} \sqrt{B_{1}}}{\sqrt{\left|\left(B_{1}^{2}-B_{2}\right)\left(1+q^{2}\right)+\left(B_{1}^{2}-2 B_{2}\right) q\right|+B_{1}\left(1+2 q+q^{2}\right)}}\right\}
$$

and

$$
\left|a_{3}\right| \leq \min \{L(q), M(q)\}
$$

where

$$
\begin{gathered}
K(q)=\left\{\begin{array}{ll}
\sqrt{\frac{B_{1}}{1+q+q^{2}} ;}, & \left|B_{2}\right| \leq B_{1} \\
\sqrt{\frac{\left|B_{2}\right|}{1+q+q^{2}} ;} & \left|B_{2}\right|>B_{1}
\end{array},\right. \\
L(q)= \begin{cases}\frac{B_{1}}{1+q+q^{2}} ; & \left|B_{2}\right| \leq B_{1} \\
\frac{\left|B_{2}\right|}{1+q+q^{2}} ; & \left|B_{2}\right|>B_{1}\end{cases}
\end{gathered}
$$

and

$$
M(q)=\left\{\begin{array}{lr}
\frac{B_{1}}{1+q+q^{2}} ; & B_{1} \leq \frac{1+2 q+q^{2}}{1+q+q^{2}} \\
\frac{B_{1}\left[\left|\left(B_{1}^{2}-B_{2}\right)\left(1+q^{2}\right)+\left(B_{1}^{2}-2 B_{2}\right) q\right|+B_{1}^{2}\left(1+q+q^{2}\right)\right]}{\left[\left|\left(B_{1}^{2}-B_{2}\right)\left(1+q^{2}\right)+\left(B_{1}^{2}-2 B_{2}\right) q\right|+B_{1}\left(1+2 q+q^{2}\right)\right]\left(1+q+q^{2}\right)} ; & B_{1}>\frac{1+2 q+q^{2}}{1+q+q^{2}}
\end{array} .\right.
$$




\section{COROLLARIES AND CONCLUDING REMARKS}

Corollary 1. If we take

$$
\phi(z)=\left(\frac{1+z}{1-z}\right)^{\alpha}=1+2 \alpha z+2 \alpha^{2} z^{2}+\ldots \quad(0<\alpha \leq 1),
$$

in Theorem 1 and Theorem 2, respectively, we have

$$
\left|a_{n}\right| \leq \frac{2 \alpha|b|}{[n]_{p, q}} ; \quad n \geq 3
$$

and

$$
\left|a_{n}\right| \leq \frac{2 \alpha|b|(1-q)}{1-q^{n}} ; \quad n \geq 3 .
$$

Remark 1. Let $f \in R_{\Sigma}^{p, q}\left(\left(\frac{1+z}{1-z}\right)^{\alpha}\right)$. Then

$$
\left|a_{n}\right| \leq \frac{2 \alpha}{[n]_{p, q}} ; \quad n \geq 3 .
$$

Remark 2. Let $f \in R_{\Sigma}^{q}\left(\left(\frac{1+z}{1-z}\right)^{\alpha}\right)$. Then

$$
\left|a_{n}\right| \leq \frac{2 \alpha(1-q)}{1-q^{n}} ; \quad n \geq 3 .
$$

Corollary 2. If we take

$$
\phi(z)=\frac{1+(1-2 \beta) z}{1-z}=1+2(1-\beta) z+2(1-\beta) z^{2}+\cdots \quad(0 \leq \beta<1),
$$

in Theorem 1 and Theorem 2, respectively, we have

and

$$
\left|a_{n}\right| \leq \frac{2(1-\beta)|b|}{[n]_{p, q}} ; \quad n \geq 3
$$

$$
\left|a_{n}\right| \leq \frac{2(1-\beta)|b|(1-q)}{1-q^{n}} ; \quad n \geq 3
$$

Remark 3. Let $f \in R_{\Sigma}^{p, q}\left(\frac{1+(1-2 \beta) z}{1-z}\right)$. Then

$$
\left|a_{n}\right| \leq \frac{2(1-\beta)}{[n]_{p, q}} ; \quad n \geq 3 .
$$

Remark 4. Let $f \in R_{\Sigma}^{p, q}\left(\frac{1+(1-2 \beta) z}{1-z}\right)$. Then

$$
\left|a_{n}\right| \leq \frac{2(1-\beta)(1-q)}{1-q^{n}} ; \quad n \geq 3 .
$$


Taking $\phi(z)=\left(\frac{1+z}{1-z}\right)^{\alpha}$ in Theorem 5 and Theorem 6, then we have the following results.

Corollary 3. Let $f \in \mathrm{R}_{\Sigma, b}^{p, q}(\phi)(b \in \mathbb{C} \backslash\{0\})$. Then

$$
\left|a_{2}\right| \leq \min \left\{\sqrt{\frac{2 \alpha|b|}{p^{2}+p q+q^{2}}}, \frac{2 \alpha|b|}{\sqrt{\alpha\left|(2 b-1)\left(p^{2}+q^{2}\right)+2(b-1) p q\right|+p^{2}+2 p q+q^{2}}}\right\}
$$

and

where

$$
\left|a_{3}\right| \leq \min \left\{\frac{2 \alpha|b|}{p^{2}+p q+q^{2}}, H(p, q)\right\}
$$

$$
H(p, q)=\left\{\begin{array}{lc}
\frac{2 \alpha|b|}{p^{2}+p q+q^{2}} ; & 0<\alpha \leq \frac{p^{2}+2 p q+q^{2}}{2\left(p^{2}+p q+q^{2}\right)|b|} \\
\frac{2 \alpha^{2}|b|\left[\left|(2 b-1)\left(p^{2}+q^{2}\right)+2(b-1) p q\right|+2|b|\left(p^{2}+p q+q^{2}\right)\right]}{\left[\alpha\left|(2 b-1)\left(p^{2}+q^{2}\right)+2(b-1) p q\right|+p^{2}+2 p q+q^{2}\right]\left(p^{2}+p q+q^{2}\right)} ; & \frac{p^{2}+2 p q+q^{2}}{2\left(p^{2}+p q+q^{2}\right)|b|}<\alpha \leq 1
\end{array} .\right.
$$

Corollary 4. Let $f \in \mathrm{R}_{\Sigma, b}^{q}(\phi)(b \in \mathbb{C} \backslash\{0\})$. Then

$$
\left|a_{2}\right| \leq \min \left\{\sqrt{\frac{2 \alpha|b|}{1+q+q^{2}}}, \frac{2 \alpha|b|}{\sqrt{\alpha\left|(2 b-1)\left(1+q^{2}\right)+2(b-1) q\right|+1+2 q+q^{2}}}\right\}
$$

and

where

$$
\left|a_{3}\right| \leq \min \left\{\frac{2 \alpha|b|}{1+q+q^{2}}, H(q)\right\}
$$

$$
H(q)=\left\{\begin{array}{lc}
\frac{2 \alpha|b|}{1+q+q^{2}} ; & 0<\alpha \leq \frac{1+2 q+q^{2}}{2\left(1+q+q^{2}\right)|b|} \\
\frac{2 \alpha^{2}|b|\left[\left|(2 b-1)\left(1+q^{2}\right)+2(b-1) q\right|+2|b|\left(1+q+q^{2}\right)\right]}{\left[\alpha\left|(2 b-1)\left(1+q^{2}\right)+2(b-1) q\right|+\left(1+2 q+q^{2}\right)\right]\left(1+q+q^{2}\right)} ; & \frac{1+2 q+q^{2}}{2\left(1+q+q^{2}\right)|b|}<\alpha \leq 1
\end{array} .\right.
$$

Corollary 5. Let $f \in \mathrm{R}_{\Sigma}^{p, q}\left(\left(\frac{1+z}{1-z}\right)^{\alpha}\right)$. Then

and

$$
\left|a_{2}\right| \leq \frac{2 \alpha}{\sqrt{(\alpha+1)\left(p^{2}+q^{2}\right)+2 p q}}
$$

where

$$
\left|a_{3}\right| \leq \min \left\{\frac{2 \alpha}{p^{2}+p q+q^{2}}, H(p, q)\right\}
$$

$$
H(p, q)=\left\{\begin{array}{lc}
\frac{2 \alpha}{p^{2}+p q+q^{2}} ; & 0<\alpha \leq \frac{p^{2}+2 p q+q^{2}}{2\left(p^{2}+p q+q^{2}\right)} \\
\frac{2 \alpha^{2}\left[3\left(p^{2}+q^{2}\right)+2 p q\right]}{\left[(\alpha+1)\left(p^{2}+q^{2}\right)+2 p q\right]\left(p^{2}+p q+q^{2}\right)} ; & \frac{p^{2}+2 p q+q^{2}}{2\left(p^{2}+p q+q^{2}\right)}<\alpha \leq 1
\end{array} .\right.
$$


Corollary 6. Let $f \in \mathrm{R}_{\Sigma}^{q}\left(\left(\frac{1+z}{1-z}\right)^{\alpha}\right)$. Then

$$
\left|a_{2}\right| \leq \frac{2 \alpha}{\sqrt{(\alpha+1)\left(1+q^{2}\right)+2 q}}
$$

and

where

$$
\left|a_{3}\right| \leq \min \left\{\frac{2 \alpha}{1+q+q^{2}}, H(q)\right\}
$$

$$
H(q)=\left\{\begin{array}{lc}
\frac{2 \alpha}{1+q+q^{2}} ; & 0<\alpha \leq \frac{1+2 q+q^{2}}{2\left(1+q+q^{2}\right)} \\
\frac{2 \alpha^{2}\left[3\left(1+q^{2}\right)+2 q\right]}{\left[(\alpha+1)\left(1+q^{2}\right)+2 q\right]\left(1+q+q^{2}\right)} ; & \frac{1+2 q+q^{2}}{2\left(1+q+q^{2}\right)}<\alpha \leq 1
\end{array} .\right.
$$

By choosing $\phi(z)=\frac{1+(1-2 \beta) z}{1-z}$ in Theorem 5 and Theorem 6 , then we have the following results.

Corollary 7. Let $f \in \mathrm{R}_{\Sigma, b}^{p, q}(\phi)(b \in \mathbb{C} \backslash\{0\})$. Then

$$
\left|a_{2}\right| \leq \min \left\{\sqrt{\frac{2(1-\beta)|b|}{p^{2}+p q+q^{2}}}, \frac{2(1-\beta)|b|}{\sqrt{\left|2(1-\beta) b[3]_{p, q}-[2]_{p, q}^{2}\right|+[2]_{p, q}^{2}}}\right\}
$$

and

$$
\left|a_{3}\right| \leq \min \left\{\frac{2(1-\beta)|b|}{p^{2}+p q+q^{2}}, R(p, q)\right\}
$$

where

$$
R(p, q)=\left\{\begin{array}{lc}
\frac{2(1-\beta)|b|}{p^{2}+p q+q^{2}} ; & \frac{2\left(p^{2}+p q+q^{2}\right)|b|-\left(p^{2}+2 p q+q^{2}\right)}{2\left(p^{2}+p q+q^{2}\right)|b|} \leq \beta<1 \\
\frac{2(1-\beta)|b|\left[\left|2(1-\beta) b\left(p^{2}+p q+q^{2}\right)-\left(p^{2}+2 p q+q^{2}\right)\right|+2(1-\beta)|b|\left(p^{2}+p q+q^{2}\right)\right]}{\left[\left|2(1-\beta) b\left(p^{2}+p q+q^{2}\right)-\left(p^{2}+2 p q+q^{2}\right)\right|+\left(p^{2}+2 p q+q^{2}\right)\right]\left(p^{2}+p q+q^{2}\right)} & 0 \leq \beta<\frac{2\left(p^{2}+p q+q^{2}\right)|b|-\left(p^{2}+2 p q+q^{2}\right)}{2\left(p^{2}+p q+q^{2}\right)|b|}
\end{array}\right.
$$

Corollary 8. Let $f \in \mathrm{R}_{\Sigma, b}^{q}(\phi)(b \in \mathbb{C} \backslash\{0\})$. Then

$$
\left|a_{2}\right| \leq \min \left\{\sqrt{\frac{2(1-\beta)|b|}{1+q+q^{2}}}, \frac{2(1-\beta)|b|}{\sqrt{\left|2(1-\beta) b\left(1+q+q^{2}\right)-\left(1+2 q+q^{2}\right)\right|+1+q+q^{2}}}\right\}
$$

and

$$
\left|a_{3}\right| \leq \min \left\{\frac{2(1-\beta)|b|}{1+q+q^{2}}, R(q)\right\}
$$


where

$$
R(q)=\left\{\begin{array}{cc}
\frac{2(1-\beta)|b|}{1+q+q^{2}} ; & \frac{2\left(1+q+q^{2}\right)|b|-\left(1+2 q+q^{2}\right)}{2\left(1+q+q^{2}\right)|b|} \leq \beta<1 \\
\frac{2(1-\beta)|b|\left[\left|2(1-\beta) b\left(1+q+q^{2}\right)-\left(1+2 q+q^{2}\right)\right|+2(1-\beta)|b|\left(1+q+q^{2}\right)\right]}{\left[\left|2(1-\beta) b\left(1+q+q^{2}\right)-\left(1+2 q+q^{2}\right)\right|+1+2 q+q^{2}\right]\left(1+q+q^{2}\right)} \\
0 \leq \beta<\frac{2\left(1+q+q^{2}\right)|b|-\left(1+2 q+q^{2}\right)}{2\left(1+q+q^{2}\right)|b|}
\end{array}\right.
$$

Corollary 9. Let $f \in \mathrm{R}_{\Sigma}^{p, q}\left(\frac{1+(1-2 \beta) z}{1-z}\right)$. Then

$$
\left|a_{2}\right| \leq \min \left\{\sqrt{\frac{2(1-\beta)}{p^{2}+p q+q^{2}}}, \frac{2(1-\beta)}{\sqrt{\left|(1-2 \beta)\left(p^{2}+q^{2}\right)-2 \beta p q\right|+p^{2}+2 p q+q^{2}}}\right\}
$$

and

$$
\left|a_{3}\right| \leq \min \left\{\frac{2(1-\beta)}{p^{2}+p q+q^{2}}, R(p, q)\right\}
$$

where

$$
R(p, q)=\left\{\begin{array}{ll}
\frac{2(1-\beta)}{p^{2}+p q+q^{2}} ; & \frac{p^{2}+q^{2}}{2\left(p^{2}+p q+q^{2}\right)} \leq \beta<1 \\
\frac{(3-4 \beta)\left(p^{2}+q^{2}\right)+2(1-2 \beta) p q}{\left(p^{2}+p q+q^{2}\right)^{2}} ; & 0 \leq \beta<\frac{p^{2}+q^{2}}{2\left(p^{2}+p q+q^{2}\right)}
\end{array} .\right.
$$

Corollary 10. Let $f \in \mathrm{R}_{\Sigma}^{q}\left(\frac{1+(1-2 \beta) z}{1-z}\right)$. Then

$$
\left|a_{2}\right| \leq \min \left\{\sqrt{\frac{2(1-\beta)}{1+q+q^{2}}}, \frac{2(1-\beta)}{\sqrt{\left.\mid(1-2 \beta)\left(1+q^{2}\right)-2 \beta q\right) \mid+1+q+q^{2}}}\right\}
$$

and

where

$$
\left|a_{3}\right| \leq \min \left\{\frac{2(1-\beta)}{1+q+q^{2}}, R(q)\right\}
$$

$$
R(q)=\left\{\begin{array}{ll}
\frac{2(1-\beta)}{1+q+q^{2}} ; & \frac{1+q^{2}}{2\left(1+q+q^{2}\right)} \leq \beta<1 \\
\frac{(3-4 \beta)\left(1+q^{2}\right)+2(1-2 \beta) q}{\left(1+q+q^{2}\right)^{2}} ; & 0 \leq \beta<\frac{1+q^{2}}{2\left(1+q+q^{2}\right)}
\end{array} .\right.
$$

\section{REFERENCES}

[1] H. Airault, Symmetric sums associated to the factorization of Grunsky coefficients. Montreal, Canada: in Conference, Groups and Symmetries, 2007.

[2] H. Airault, "Remarks on Faber polynomials," Int. Math. Forum, vol. 3, pp. 449-456, 2008.

[3] H. Airault and H. Bouali, "Differential calculus on the Faber polynomials," Bulletin des Sciences Mathematiques, pp. 179-222, 2006, doi: 10.1016/j.bulsci.2005.10.002. 
[4] H. Airault and J. Ren, "In algebra of differential operators and generating functions on the set of univalent functions," Bulletin des Sciences Mathematiques, vol. 126, pp. 343-367, 2002, doi: 10.1016/S0007-4497(02)01115-6.

[5] Ş. Altınkaya and S. Yalçın, "Coefficient bounds for a subclass of bi-univalent functions,", vol. 6, no. 2, pp. 180-185, 2015.

[6] Ş. Altınkaya and S. Yalçın, "Faber polynomial coefficient bounds for a subclass of biunivalent functions," C.R. Acad. Sci. Paris, Ser.I, vol. 353, no. 12, pp. 1075-1080, 2015, doi: 10.1016/j.crma.2015.09.003.

[7] D. A. Brannan and J. G. Clunie, Aspects of contemporary complex analysis. New York: Proceedings of the NATO Advanced Study Instute Held at University of Durham, 1979.

[8] D. A. Brannan and T. S. Taha, "On some classes of bi-univalent functions," Studia Universitatis Babeş-Bolyai Mathematica, vol. 31, no. 2, pp. 70-77, 1986.

[9] T. Bulboaca and P. Goswami, "On some classes of bi-univalent functions," Studia Universitatis Babeş-Bolyai Mathematica, vol. 2, no. 2, pp. 7-13, 2015.

[10] R. Chakrabarti and R. Jagannathan, "A $(p, q)$-oscillator realization of two-parameter quantum algebras,” J. Phys. A: Math. Gen, vol. 24, pp. L711-L718, 1991.

[11] P. Duren, Univalent Functions. New York: Grundlehren der Mathematischen Wissenschaften, Springer, 1983.

[12] G. Faber, "Über polynomische entwickelungen,” Math. Ann, vol. 57, pp. 1569-1573, 1903.

[13] G. Gasper and M. Rahman, Basic Hypergeometric Series. Cambridge: Cambridge Univ. Press, 1990.

[14] H. Grunsky, "Koeffizientenbedingungen für schlicht abbildende meromorphe funktionen," Math. Zeit, vol. 45, pp. 29-61, 1939.

[15] S. G. Hamidi and J. M. Jahangiri, "Faber polynomial coefficient estimates for analytic biclose-to-convex functions," C. R. Acad. Sci. Paris, Ser.I, vol. 352, pp. 17-20, 2014, doi: 10.1016/j.crma.2013.11.005.

[16] S. G. Hamidi and J. M. Jahangiri, "Faber polynomial coefficients of bi-subordinate functions," $C$. R. Acad. Sci. Paris, Ser.I, vol. 354, pp. 365-370, 2016, doi: 10.1016/j.crma.2016.01.013.

[17] T. Hayami and S. Owa, "Coefficient bounds for bi-univalent functions," Pan Amer. Math, vol. 22, no. 4, pp. 15-26, 2012.

[18] F. H. Jackson, "On $q$-functions and a certain difference operator," Appl. Math. Sci, vol. 46, no. 2, pp. 253-281, 1908, doi: 10.1017/S0080456800002751.

[19] M. Lewin, "On a coefficient problem for bi-univalent functions," Proc. Amer. Math. Soc, vol. 18, no. 1 , pp. 63-68, 1967, doi: 10.2307/2035225.

[20] E. Netanyahu, "The minimal distance of the image boundary from the origin and the second coefficient of a univalent function in $|z|<1$," Archive for Rational Mechanics and Analysis, vol. 32, pp. 100-112, 1969.

[21] A. C. Schaeffer and D. C. Spencer, "The coefficients of schlicht functions," Duke Math. J, vol. 10, pp. 611-635, 1943.

[22] M. Schiffer, "A method of variation within the family of simple functions," Proc. London Math. Soc, vol. 44, pp. 432-449, 1938.

[23] B. Şeker and V. Mehmetoğlu, "Coefficient bounds for new subclasses of bi-univalent functions," New Trends in Mathematical Sciences, vol. 4, no. 3, pp. 197-203, 2016, doi: 10.20852/ntmsci.2016318930.

[24] H. M. Srivastava, A. K. Mishra, and P. Gochhayat, "Certain subclasses of analytic and bi-univalent functions," Applied Mathematics Letters, vol. 23, pp. 1188-1192, 2010, doi: 10.1016/j.aml.2010.05.009. 
[25] H. M. Srivastava, G. Murugusundaramoorthy, and N. Magesh, "Certain subclasses of bi-univalent functions associated with the Hohlov operator," Applied Mathematics Letters, vol. 1, no. 2, pp. 6773, 2013, doi: Certain subclasses of bi-univalent functions associated with the Hohlov operator.

\section{Authors' addresses}

\section{Şahsene Altınkaya}

Uludag University, Department of Mathematics, Faculty of Arts and Science, 16059 Bursa, Turkey

E-mail address: sahsene@uludag.edu.tr

\section{Sibel Yalçın}

Uludag University, Department of Mathematics, Faculty of Arts and Science, 16059 Bursa, Turkey

E-mail address: syalcin@uludag.edu.tr 